\title{
LA FORMACIÓN DOCENTE EN LA SOCIEDAD DEL CONOCIMIENTO Y LA INFORMACIÓN: AVANCES $Y$ TEMAS PENDIENTES*
}

\section{TEACHER EDUCATION IN THE KNOWLEDGE AND INFORMATION SOCIETY: ADVANCEMENTS AND PENDING THEMES ${ }^{* *}$}

Carlos Marcelo GARCÍA***

\begin{abstract}
Resumo: Nuestras sociedades están envueltas en un complicado proceso de transformación. Una transformación no planificada que está afectando a la forma como nos organizamos, cómo trabajamos, cómo nos relacionamos, y cómo aprendemos. Estos cambios tienen un reflejo visible en la escuela como institución encargada de formar a los nuevos ciudadanos. ¿En qué afectan estos cambios a los profesores? ¿Cómo debemos repensar el trabajo del profesor en estas nuevas circunstancias? ¿Cómo deberían formarse los nuevos profesores? ¿Cómo adecuamos los conocimientos y las actitudes del profesorado para dar respuesta y aprovechar las nuevas oportunidades que la sociedad de la información nos ofrece? En este artículo revisamos algunos avances y temas pendientes que la formación tiene para enfrentarse con eficiencia a las necesidades de la sociedad del conocimiento: universidad y escuela: la laguna de los dos mundos; formadores de profesores universitarios y profesores supervisores de aula; conocimiento disciplinar y conocimiento pedagógico; teoría y práctica: desvalorización de la teoría y adoración de la práctica; tradición e innovación; homogeneidad y
\end{abstract}

\footnotetext{
* Ponencia presentada al IV Encuentro Internacional de KIPUS. Políticas públicas y formación docente, Isla Margarita (Venezuela, 4-6 de octubre, 2006).

${ }^{* *}$ Paper presented at the IV International Meeting - KIPUS. Public policies and teacher education, Isla Margarita, (Venezuela, 4-6th October 2006).

${ }^{* * *}$ Professor da Universidad de Sevilla. E-mail: marcelo@us.es
} 
La formación docente en la sociedad del conocimiento y la información

diversidad: profesores homogéneos para un alumnado diverso; enseñanza y aprendizaje; formación inicial y formación continua; aprendizaje formal y informal; aislamiento en el aula y la sociedad en red.

Palabras claves: Formación docente. Sociedad del Conocimiento. Aprender a Enseñar

\begin{abstract}
Our societies are involved in a complex transformation process. An unplanned transformation that is affecting the way we organize ourselves, how we work, how we relate to others and how we learn. These changes have a clear impact on schools as they are the institutions responsible for educating the new citizens. How do these changes affect teachers? How should we rethink teachers' work under these new circumstances? How should the new teachers be educated? How do we adapt teachers' knowledge and attitudes so that they take advantage of the new opportunities that the information society offers them? In this article we consider some of the advancements and other pending themes that teacher education has in order to face with efficiency the information society needs: university and school, the gap between the two worlds, educators of university professors and teachers who supervise classes. Content knowledge and pedagogical knowledge. Theory and practice: depreciation of theory and an excessive valorization of practice. Tradition and innovation. Homogeneity and diversity: homogeneous teachers for heterogeneous groups of students.
\end{abstract}

Keywords: Teacher education. knowledge society. learning to teach.

\title{
INTRODUCCIÓN: LOS PROFESORES CUENTAN
}

Nuestras sociedades están envueltas en un complicado proceso de transformación. Una transformación no planificada que está afectando a la forma como nos organizamos, cómo trabajamos, cómo 
nos relacionamos, y cómo aprendemos. Estos cambios tienen un reflejo visible en la escuela como institución encargada de formar a los nuevos ciudadanos. Por poner un ejemplo, nuestros alumnos disponen hoy en día de muchas más fuentes de información que lo que ocurría no hace ni diez años. Fuentes de información que, aportadas por las nuevas tecnologías de la información y comunicación, están haciendo necesario un replanteo de las funciones que tradicionalmente se han venido asignando a las escuelas y a los profesionales que en ella trabajan: los profesores y profesoras.

Una de las características de la sociedad en la que vivimos tiene que ver con que el conocimiento es uno de los principales valores de sus ciudadanos. El valor de las sociedades actuales está directamente relacionado con el nivel de formación de sus ciudadanos, y de la capacidad de innovación y emprendimiento que estos posean. Pero los conocimientos, en nuestros días, tienen fecha de caducidad y ello nos obliga ahora más que nunca a establecer garantías formales e informales para que los ciudadanos y profesionales actualicen constantemente su competencia. Hemos entrado en una sociedad que exige de los profesionales una permanente actividad de formación y aprendizaje.

¿En qué afectan estos cambios a los profesores? ¿Cómo debemos repensar el trabajo del profesor en estas nuevas circunstancias? ¿Cómo deberían formarse los nuevos profesores? ¿Cómo adecuamos los conocimientos y las actitudes del profesorado para dar respuesta y aprovechar las nuevas oportunidades que la sociedad de la información nos ofrece?

Las preguntas anteriores configuran todo un programa de preocupaciones que está llevando a muchos académicos, profesionales, investigadores, docentes, etc. a pensar en que la escuela tiene que dar respuesta pronta a los desafíos que se le avecinan. Respuestas que van directamente relacionadas con la capacidad de ofrecer la mejor educación para todos los alumnos. Y para ello volvemos la vista hacia el profesorado que trabaja codo a codo con nuestros estudiantes. ¿Cómo se han formado? ¿Qué cambios hay que introducir en su formación para que sean de nuevo los líderes de un cambio que la sociedad está demandando? (MARCELO, 2002a).

Recientes informes internacionales han venido a centrarse y a destacar el importante papel que el profesorado juega en relación con las posibilidades de aprendizaje de los alumnos. Ya el mismo título de informe que la OCDE ha publicado recientemente nos llama la atención: 
La formación docente en la sociedad del conocimiento y la información

"Teachers matter: attracting, developing and retaining effective teachers" (OCDE, 2005). Se afirma en el título que los profesores cuentan, importan para ayudar a mejorar la calidad de la enseñanza que reciben los alumnos. Se afirma en este informe que:

Existe actualmente un volumen considerable de investigación que indica que la calidad de los profesores y de su enseñanza es el factor más importante para explicar los resultados de los alumnos. Existen también considerables evidencias de que los profesores varían en su eficacia. Las diferencias entre los resultados de los alumnos a veces son mayores dentro de la propia escuela que entre escuelas. La enseñanza es un trabajo exigente, y no es posible para cualquiera ser un profesor eficaz y mantener esta eficacia a lo largo del tiempo (p. 12).

Este informe viene a mostrar la preocupación internacional en relación con el profesorado, con las formas de hacer de la docencia una profesión atractiva, con cómo mantener en la enseñanza a los mejores profesores y cómo conseguir que los profesores sigan aprendiendo a lo largo de su carrera.

Este informe de la OCDE viene a mostrar que el profesorado cuenta. Cuenta para influir en el aprendizaje de los alumnos. Cuenta para mejorar la calidad de la educación que las escuelas e institutos llevan a cabo día a día. Cuenta en definitiva como una profesión necesaria e imprescindible para la sociedad del conocimiento. Y puesto que el profesorado cuenta, necesitamos que nuestros sistemas educativos sean capaces de atraer a los mejores candidatos para convertirse en docentes. Necesitamos buenas políticas para que la formación inicial de estos profesores les asegure las competencias que van a requerir a lo largo de su extensa, flexible y variada trayectoria profesional. Y la sociedad necesita buenos profesores cuya práctica profesional cumpla los estándares profesionales de calidad que asegure el compromiso de respetar el derecho que los alumnos tienen de aprender.

Paralelamente al estudio de la OCDE, la prestigiosa Asociación Americana de Investigación Educativa (A.E.R.A.) ha hecho público el informe que intenta resumir los resultados de la investigación sobre la formación del profesorado, así como hacer propuestas de política educativa acordes con estos resultados. Se afirma que: "en toda la nación existe un consenso emergente acerca de que el profesorado influye de 
manera significativa en el aprendizaje de los alumnos y en la eficacia de la escuela" (COCHRAN-SMITH; FRIES, 2005, p. 40). En la misma línea, Daling-Hammond (2000) venía afirmar que el aprendizaje de los alumnos "depende principalmente de lo que los profesores conocen y de lo que pueden hacer”.

\section{LA DOCENCIA ANTE LOS CAMBIOS}

A la tarea de enseñar los profesores se enfrentan generalmente en solitario. Sólo los alumnos son testigos de la actuación profesional de los profesores. Pocas profesiones se caracterizan por una mayor soledad y aislamiento. A diferencia de otras profesiones u oficios, la enseñanza es una actividad que se desarrolla en solitario. Como de forma acertada afirmara Bullough (1998), la clase es el santuario de los profesores.El santuario de la clase es un elemento central de la cultura de la enseñanza, que se preserva y protege mediante el aislamiento, y que padres, directores y otros profesores dudan en violar. Cuando hoy en día estamos asistiendo a propuestas que evidentemente plantean la necesidad de que los profesores colaboren, trabajen conjuntamente, etc., nos encontramos con la pertinaz realidad de profesores que se refugian en la soledad de sus clases. Ya resulta clásico el estudio llevado a cabo por Lortie en 1975, en el que mediante entrevistas estableció algunas características de la profesión docente en Estados Unidos, que no sólo son de gran actualidad, sino que son perfectamente aplicables a nuestro país. Una característica identificada por Lortie fue el Individualismo. Este individualismo se produce en opinión del autor por la ausencia de ocasiones en las que los profesores puedan observarse unos a otros, y ello se produce desde los primeros años de formación como profesor y posteriormente durante el proceso de socialización.

El aislamiento de los profesores está favorecido evidentemente por la arquitectura escolar, que organiza la escuela en módulos estándar, así como por la distribución del tiempo y el espacio, y la existencia de normas de independencia y privacidad entre los profesores. El aislamiento, como norma y cultura profesional tiene ciertas ventajas y algunos evidentes inconvenientes para los profesores. El aislamiento representa una barrera real frente a las posibilidades de formación y de mejora. Los cambios que se están produciendo en la sociedad inciden en la demanda de una "redefinición del trabajo del profesor" y seguramente de la 
profesión docente, de su formación y de su desarrollo profesional. Los roles que tradicionalmente han asumido los docentes enseñando de manera conservadora un curriculum caracterizado por contenidos académicos hoy en día resultan a todas luces inadecuados. A los alumnos les llega la información por múltiples vías: la televisión, radio, ordenadores, Internet, recursos culturales de las ciudades, etc. Y los profesores no pueden hacer como si nada de esto fuera con ellos. Salomon (1992) nos ofrecía su metáfora respecto a que se está modificando el rol del profesor desde transmisor de información, el solista de una flauta al frente de una audiencia poco respetuosa, al de un diseñador, un guía turístico, un director de orquesta. Así, el papel del profesor debería de cambiar desde una autoridad que distribuye conocimientos hacia un sujeto que crea y orquesta ambientes de aprendizaje complejos, implicando a los alumnos en actividades apropiadas, de manera que los alumnos puedan construir su propia comprensión del material a estudiar, trabajando con los alumnos como compañeros en el proceso de aprendizaje.

Cuando estamos viendo día a día las nuevas exigencias que las escuelas y el profesorado están recibiendo por parte de la sociedad, asistimos a una situación en la observamos que la profesión docente puede que no esté asumiendo la responsabilidad que le corresponde como profesión del conocimiento. La creciente presión por una escuela de mayor calidad, motivada en parte por los resultados de los informes internacionales, no está teniendo su contrapartida en una profesión docente que lidere y canalice los cambios que tanto dentro de las aulas como en la escuela y en la formación del profesorado se necesitan para que las escuelas sigan siendo espacios privilegiados de socialización de las nuevas generaciones.

¿Qué hay del profesorado y de su formación? ¿Qué cambios observamos en la dirección correcta? ¿Podemos hablar de que la formación esté girando hacia las necesidades de la sociedad del conocimiento y la información? Veamos algunas ideas al respecto.

\section{LA FORMACIÓN DEL PROFESORADO EN LA SOCIEDAD DEL CONOCIMIENTO: AVANCES}

Los informes internacionales anteriormente referidos ponen de manifiesto de nuevo que la influencia del profesorado es determinante. Y si esto es así, hoy en día más que nunca debemos de atender a las 
estrategias y procesos que permiten que el profesorado alcance y mantenga niveles de competencia y eficacia elevados. Y ello no es posible si no disponemos de un sistema de formación que ayude a reclutar, formar, insertar y desarrollar al profesorado a lo largo de toda su carrera docente. Hemos dicho que en las sociedades actuales el conocimiento y la formación configuran elementos estratégicos para el desarrollo de las personas y de los países. Ello es así ahora más que nunca. Poco a poco ha venido haciéndose familiar la idea de que el aprendizaje es para toda la vida. Hoy en día ya es una realidad para todo el profesorado.

Necesitamos formación pero no cualquier formación. Estos cambios nos están dirigiendo a pensar que la formación no puede mantenerse en los estándares pasados ni actuales. Carl Bereiter (2002) decía que para educar en la era del conocimiento necesitamos una nueva idea de la mente. Una idea o concepción que se aleje de entender la mente como un contenedor y que la entienda más bien como un sistema que se autorregula y organiza a partir de múltiples conexiones. Pues bien, creo que también debemos de pensar en la formación de una forma más abierta y flexible que se adapte a las necesidades de los individuos y que permita no sólo aprender a enseñar sino generar conocimiento e innovación sobre la enseñanza que pueda ser validado y compartido.

Para avanzar en este objetivo no empezamos de cero. Para responder a estas preguntas no partimos de cero. Cómo se aprender a enseñar ha sido una constante en la preocupación de los investigadores educativos en las últimas décadas. Cientos de investigaciones y decenas de revisiones se han llevado a cabo para intentar comprender este proceso. Tanto en el tercer como en el cuarto "Handbook of Research on Teaching" (RICHARDSON, 2001) encontramos capítulos en los que se revisa y sintetiza el conocimiento sobre los profesores, su formación y desarrollo. Igualmente en los "Handbook of Research on Teacher Education", pasando por el "International Handbook of Teachers and Teaching", el "International Handbook of Educational Change", o el "Handbook of Educational Psychology" se aborda de manera más o menos amplia la investigación sobre el aprendizaje de los profesores. Estos libros, así como revisiones aparecidas en revistas especializadas, como la de Wilson y Berne (1999), Feiman (2001), Putnam y Borko (1998), Wideen, Mayer-Smith y Moon (1998) o Zeichner (1999) y Cochran-Smith y Zeichner (2005) nos permiten configurar un panorama bastante actualizado respecto al conocimiento 
acumulado sobre el proceso de aprender a enseñar, así como de sus luces y sombras. A partir de estas amplias revisiones uno puede situarse y encontrar conocimiento acumulado suficiente para poder empezar a dar respuesta a la pregunta ¿cómo se aprende a enseñar? Dado que no puede ser intención de éste que escribe, resumir lo que en centenares de páginas, otros investigadores ya han revisado, y debido a la concreción necesaria de esta escrito, vamos a hacer un recorrido acerca de los aspectos básicos que dan respuesta a la intención del título.

Convertirse en profesor es un largo proceso. A las instituciones de formación inicial del profesorado llegan candidatos que no son "vasos vacíos". Como ya investigara Lortie (1975), las miles de horas de observación como estudiantes contribuyen a configurar un sistema de creencias hacia la enseñanza que los aspirantes a profesores tienen y que les ayudan a interpretar sus experiencias en la formación. Estas creencias a veces están tan arraigadas que la formación inicial no consigue el más mínimo cambio profundo en ellas (PAJARES, 1992; RICHARDSON; PLACIER, 2001).

La formación inicial del profesorado ha sido objetivo de múltiples estudios e investigaciones (COCHRAN-SMITH; FRIES, 2005). En general se observa una gran insatisfacción tanto de las instancias políticas como del profesorado en ejercicio o de los propios formadores respecto a la capacidad de las actuales instituciones de formación para dar respuesta a las necesidades actuales de la profesión docente. Las críticas hacia su organización burocratizada, el divorcio entre la teoría y la práctica, la excesiva fragmentación del conocimiento que se enseña, la escasa vinculación con las escuelas (FEIMAN-NEMSER, 2001) están haciendo que ciertas voces críticas propongan reducir la extensión de la formación inicial para incrementar la atención al periodo de inserción del profesorado en la enseñanza. Es el caso del reciente informe de la OCDE al que ya hemos hecho referencia anteriormente. En concreto, se afirma que

Las etapas de formación inicial, inserción y desarrollo profesional deberían de estar mucho más interrelacionadas para crear un aprendizaje coherente y un sistema de desarrollo para los profesores...Una perspectiva de aprendizaje a lo largo de la vida para los profesores implica para la mayoría de los países una atención más destacada a ofrecer apoyo a los profesores en sus primeros años de enseñanza, y en proporcionarles incentivos y recursos para su desarrollo profesional continuo. En general, sería más adecuado mejorar la inserción y el desarrollo profesional de 
los profesores a lo largo de su carrera en lugar de incrementar la duración de la formación inicial. (OCDE, 2005, p. 13).

Frente a estas propuestas, viene bien recordar el excelente artículo escrito por David Berliner (2000) en el que refuta una docena de críticas que habitualmente se hacen a la formación inicial del profesorado (que para enseñar basta con saber la materia, que enseñar es fácil, que los formadores de profesores viven en una torre de marfil, que los cursos de metodología y didáctica son asignaturas blandas, que en la enseñanza no hay principios generales válidos, etc. Críticas, desde el punto de vista del autor, interesadas y con una visión bastante estrecha de la contribución que la formación inicial tiene en la calidad del profesorado. Dice Berliner: "creo que se ha prestado poca atención al desarrollo de aspectos evolutivos del proceso de aprende a enseñar, desde la formación inicial, la inserción a la formación continua" ( $p$. 370). En este proceso la formación inicial juega un papel importante y no baladí o sustituible como algunos grupos o instituciones están sugiriendo.

Los profesores, en su proceso de aprendizaje, pasan por diferentes etapas momentos. Bransford, Darling-Hammond, \& LePag (2005) han planteado que para dar respuesta a las nuevas y complejas situaciones con las que se encuentran los docentes es conveniente pensar en los profesores como "expertos adaptativos" es decir personas preparadas para un aprendizaje eficiente a lo largo de toda la vida. Esto es así porque las condiciones de la sociedad son cambiantes y cada más se requiere personas que sepan combinar la competencia con la capacidad de innovación.

Los profesores principiantes necesitan poseer un conjunto de ideas y habilidades críticas así como la capacidad de reflexionar, evaluar y aprender sobre su enseñanza de tal forma que mejoren continuamente como docentes. Ello es posible si el conocimiento esencial para los profesores principiantes se pudiera organizar, representar y comunicar de forma que les permita a los alumnos una comprensión más profunda del contenido que aprenden (VAILLANT; MARCELO, 2001).

En relación a este aspecto, las investigaciones han buscado establecer diferencias entre profesores en función de la edad, así como de lo que se ha denominado "expertise". Y esta evolución, salvo en casos excepcionales, se ha comenzado a analizar a partir del primer año de experiencia docente. Por una parte tenemos aquellos estudios que intentan comprender el proceso de convertirse en experto, y por 
otro aquellos estudios que analizan qué hacen y qué caracteriza a los profesores expertos. Dentro de estos estudios ha sido clásico el contraste entre los profesores expertos y principiantes. Hay que señalar que cuando hablamos del profesor experto nos referimos no sólo a un profesor con, al menos, cinco años de experiencia docente, sino sobre todo a una persona con un "elevado nivel de conocimiento y destreza, cosa que no se adquiere de forma natural, sino que requiere una dedicación especial y constante" (Bereiter \& Scardamalia, 1986, p. 10). Así, la competencia profesional del profesor experto no se consigue a través del mero transcurrir de los años. No es totalmente cierto, como señala Berliner, que la simple experiencia sea el mejor profesor. Si no se reflexiona sobre la conducta no se llegará a conseguir un pensamiento y conducta experta (BERLINER, 1986).

Según Bereiter y Scardamalia (1986), los sujetos expertos -en cualquiera de las áreas- tienen en común las siguientes características: complejidad de las destrezas, es decir, el experto realiza sus acciones apoyándose en una estructura diferente y más compleja que la del principiante, ejerciendo un control voluntario y estratégico sobre las partes del proceso, que se desarrolla más automáticamente en el caso del principiante. En segundo lugar, figura la cantidad de conocimiento que el experto posee en relación al principiante, que posee menos conocimientos. En tercer lugar señalan la estructura del conocimiento. Para Bereiter y Scardamalia,

los principiantes tienden a tener lo que podemos describir como una estructura de conocimiento 'superficial', unas pocas ideas generales y un conjunto de detalles conectados con la idea general, pero no entre sí. Los expertos, por otra parte, tienen una estructura de conocimiento profunda y multinivel, con muchas conexiones inter e intranivel. (1986, p. 12).

La última característica que diferencia a expertos de principiantes es la representación de los problemas: el sujeto experto atiende a la estructura abstracta del problema y utiliza una variedad de tipos de problemas almacenados en su memoria. Los principiantes, por el contrario están influidos por el contenido concreto del problema y, por tanto, tienen dificultades para representarlo de forma abstracta (MARCELO, 1999b).

Conocemos por lo tanto que los profesores expertos notan e identifican las características de problemas y situaciones que pueden 
escapar la atención de los principiantes. El conocimiento experto consiste en mucho más que un listado de hechos desconectados acerca de determinada disciplina. Por el contrario, su conocimiento está conectado y organizado en torno a ideas importantes acerca de sus disciplinas. Esta organización del conocimiento ayuda a los expertos a saber cuándo, porqué, y cómo utilizar el vasto conocimiento que poseen en una situación particular.

Bransford, Derry, Berliner, \& Hammersness (2005) han planteado la necesidad de establecer una diferencia entre el "experto rutinario" y el "experto adaptativo" Ambos son expertos que siguen aprendiendo a lo largo de sus vidas. El experto rutinario desarrolla un conjunto de competencias que aplica a lo largo de su vida cada vez con mayor eficiencia. Por el contrario, el experto adaptativo tiene mayor disposición a cambiar sus competencias para profundizarlas y ampliarlas continuamente. Estos autores plantean una idea que desde mi punto de vista es bien interesante de cara a entender el proceso de inserción profesional y como consecuencia programar acciones formativas para los profesores principiantes.

\section{EL CONOCIMIENTO Y LAS CREENCIAS SE CONSTRUYEN}

Hemos constatado tanto por las investigaciones desarrolladas como por la experiencia práctica que los profesores, al igual que otras personas orientan su conducta a partir del conocimiento y creencias que poseen. Y este conocimiento y creencias se empieza a construir mucho antes que el profesor en formación decida dedicarse profesionalmente a la enseñanza. Estos conocimientos y creencias que los profesores en formación traen consigo cuando inician su formación inicial afectan de una manera directa a la interpretación y valoración que los profesores hacen de las experiencias de formación del profesorado. Esta modalidad de "aprender a enseñar" se produce a través de lo que se ha denominado "aprendizaje por la observación". Aprendizaje que en muchas ocasiones no se produce de manera intencionada, sino que se va adentrando en las estructuras de cognitivas -y emocionales- de los futuros profesores de manera inconsciente, llegando a crear expectativas y creencias difíciles de remover.

Pero al igual que desarrollamos conocimientos y creencias generales acerca de la enseñanza, de los alumnos, la escuela o el profesor, 
la materia que enseñamos o pretendemos enseñar no se queda al margen de nuestras concepciones. La forma como conocemos una determinada disciplina o área curricular afecta a cómo la enseñamos. Existen múltiples evidencias que nos muestran ciertos "arquetipos" que los profesores en formación tienen sobre la disciplina que estudian, ya sea ésta matemáticas, lengua o educación física. Preguntas como ¿qué son y para qué sirven las matemáticas, lengua, educación física, etc.? Son necesarias de plantear cuando pretendemos "partir de lo que el alumno ya sabe". Tomando el contenido que se enseña y se aprende como argumento de la indagación, podemos encontrar diferencias en el comportamiento observable de profesores en función del dominio que posean del contenido que enseñan. Existen múltiples ejemplos de investigaciones que muestran que poseer un dominio profundo de una disciplina lleva a una actividad docente más centrada en problemas, con mayor participación de los alumnos, menores digresiones y preguntas de alto orden cognitivo.

Junto al conocimiento del contenido, aprender a enseñar supone adquirir conocimiento sobre cómo se enseña la materia. Es lo que Shulman denominó "Conocimiento Didáctico del Contenido". El "Conocimiento Didáctico del Contenido" aparece como un elemento central del conocimiento del profesor. Representa la combinación adecuada entre el conocimiento de la materia a enseñar y el conocimiento pedagógico y didáctico referido a cómo enseñarla. En los últimos años, se ha venido trabajando en diferentes contextos educativos para ir clarificando cuáles son los componentes y elementos de este tipo de conocimiento profesional de la enseñanza (MARCELO, 2002a). El "Conocimiento Didáctico del Contenido" nos dirige a un debate con relación a la forma de organización, de representación, del conocimiento a través de analogías y metáforas. Plantea la necesidad de que los profesores en formación adquieran un conocimiento experto del contenido a enseñar, para que puedan desarrollar una enseñanza que propicie la comprensión de los alumnos.

La preocupación por el conocimiento como objeto de trabajo e indagación en la formación inicial del profesorado nos está conduciendo a cuestionarnos qué conocimiento es más relevante para aprender a enseñar, así como la manera en que organizamos los procesos de aprender a enseñar. Fernstermacher (1994) se preguntaba acerca de la calidad y validez epistémica del conocimiento pedagógico y didáctico, práctico y personal, que se ha venido generando en torno a la investigación sobre aprender a enseñar. No vamos a incidir en sus argumentos. Sin 
embargo, un hallazgo importante -aunque pueda resultar nimio- es que los profesores, ya sean experimentados o en formación interpretan las situaciones de enseñanza a través de las lentes que les proporcionan sus conocimientos e ideas previas. A la luz de esta afirmación, Putnam y Borko llegan a afirmar que "para la formación inicial ello significa la necesidad de atender adecuadamente al conocimiento y creencias que los profesores en formación traen consigo, creencias y conocimientos adquiridos a lo largo de su propia experiencia como estudiantes". (1998, p. 1236).

A este respecto, uno de los dilemas aun no resueltos en la formación inicial del profesorado es el que se establece entre considerar que el conocimiento que los profesores necesitan básicamente es disciplinar y aquellos que optan por la necesidad de dar mayor énfasis al conocimiento pedagógico y práctico. Bereiter (2002) plantea que en el debate sobre el currículo de la formación del profesorado, hoy en día cobra mucho más sentido recoger las propuestas que se han venido haciendo desde el movimiento que se ha denominado como "Enseñanza para la comprensión" (STONE WISKE, 1999). Este movimiento entronca con la línea de investigación sobre conocimiento didáctico del contenido y merecería una mirada más atenta en la formación del profesorado.

\section{EL CONOCIMIENTO SE CONSTRUYE EN INTERACCIÓN SOCIAL}

Por otra parte, se ha venido entendiendo que la formación y el aprendizaje del profesor pueden producirse, como hasta ahora hemos comentado, de forma relativamente autónoma y personal. Pero poco a poco ha ido ganando terreno las teorías que entienden la formación como un proceso que ocurre no de forma aislada sino dentro de un espacio intersubjetivo y social. Así, ha ido avanzando la idea de que aprender a enseñar no debería entenderse sólo como un fenómeno aislado, sino básicamente como una experiencia que ocurre en interacción con un contexto o ambiente con el que el individuo interacciona. Es la tesis del enfoque sociocultural del aprendizaje que establece que la actividad cognitiva del individuo no puede estudiarse sin tener en cuenta los contextos relacionales, sociales y culturales en que se lleva a cabo.

Está resultando de gran interés y proyección este enfoque, puesto que pone de manifiesto que la unidad de análisis del proceso de apren- 
La formación docente en la sociedad del conocimiento y la información

der a enseñar son los procesos de interacción social, llamando la atención al análisis conversacional. Así, los grupos sociales crean lo que se ha venido en llamar "comunidades discursivas" que comparten formas de pensar y de comunicarse. Comunidades que establecen redes y que sirven para compartir, intercambiar, situarse en el mundo, recibir apoyo, etc. Las comunidades de aprendizaje organizadas en torno a redes presenciales o virtuales están configurando una alternativa muy interesante a los programas tradicionales de formación.

\section{EL CONOCIMIENTO TIENE UN CARÁCTER SITUADO}

Completando la idea anterior, se ha venido avanzando en entender que el conocimiento en general y el pedagógico en particular no puede comprenderse al margen del contexto en el que surge y al que se aplica. McLellan (1996) afirma que "el modelo de conocimiento situado se basa en el principio de que el conocimiento está situado contextualmente, y está influido fundamentalmente por la actividad, el contexto y la cultura en la que se utiliza” (1996, p. 6). No cabe, por tanto, diferenciar de manera radical el conocimiento que se adquiere y el contexto en el que ese conocimiento se utiliza, de forma que el conocimiento sobre la enseñanza no puede aprenderse de forma independiente de las situaciones en las que éste se utiliza. Como consecuencia de entender el conocimiento de manera contextualizada se nos plantea con dureza la pregunta de iqué utilidad tiene para la formación inicial del profesorado un conocimiento expresado de forma proposicional, sin vínculos con la situación o contexto donde pueda contrastarse o aplicarse?

Hablamos por tanto de la capacidad de transferencia de aprendizaje que nuestros profesores en formación tienen de los conocimientos que la institución de formación considera básicos para aprender a enseñar. También nos plantea la necesidad de revisar la forma como se presenta, comunica y construye ese conocimiento. En este sentido hemos avanzado en incorporar en nuestros programas de formación de profesorado múltiples ocasiones a través de las cuales los profesores o futuros profesores pueden reflexionar sobre la enseñanza a partir del análisis de situaciones reales o simuladas. Por otra parte, la incorporación de la metodología del caso ha supuesto todo un esfuerzo por traer a las aulas de formación segmentos de la realidad de la enseñanza para que puedan ser analizados y valorados. 


\section{EL CONOCIMIENTO ESTÁ DISTRIBUIDO}

Una última característica del conocimiento que caracteriza el aprender a enseñar, es que no reside en una sola persona, sino que está distribuido, entre individuos, grupos y ambientes simbólicos y físicos (PUTNAM; BORKO, 1998). Se asume la idea de que para el desarrollo de tareas complejas, y aprender a enseñar evidentemente lo es, ninguna persona posee la totalidad de conocimientos y habilidades de forma individual.

Admitir este principio nos lleva a entender que es el trabajo en equipo lo que conduce a un mejor uso del conocimiento, lo que lleva a mejorar la capacidad de resolución de problemas. Como Senge plantea en su sugerente libro titulado "La Quinta Disciplina":

Ya no basta con tener una persona que aprenda para la organización... Ya no es posible "otear el panorama" y ordenar a los demás que sigan las órdenes del "gran estratega". Las organizaciones que cobrarán relevancia en el futuro serán las que descubran cómo aprovechar el entusiasmo y la capacidad de aprendizaje de la gente en todos los niveles de la organización. (SENGE, 1992).

La idea del conocimiento distribuido se ha visto impulsada por el impacto de las Nuevas Tecnologías, principalmente Internet. La posibilidad de que los profesores puedan acceder a conocimientos y contactos personales con profesores distantes geográficamente, la posibilidad de pertenencia a "comunidades virtuales" está ampliando las posibilidades de lo que se entiende por aprender a enseñar.

\section{LA FORMACIÓN DEL PROFESORADO EN LA SOCIEDAD DEL CONOCIMIENTO: TEMAS PENDIENTES}

A pesar de los avances que hemos intentado destacar en el epígrafe anterior, quedan pendientes algunos "temas" que parecen estar marcados a sangre y fuego en la realidad y práctica de la formación del profesorado. Vamos a comentar brevemente cuáles son estos temas que desde mi punto de vista necesitan un esfuerzo adicional. Y presento estos temas pendientes en la necesidad de superar un cierto número de yuxtaposiciones o dicotomías: 
La formación docente en la sociedad del conocimiento y la información

\section{Universidad y escuela: la laguna de los dos mundos}

Ya Feiman y Buchman (1988) llamaron la atención en relación con esta yuxtaposición. Se referían al divorcio que existe en la formación inicial según la cual, suele ocurrir que los estudiantes perciben que tanto los conocimientos, como las normas de actuación en la Institución de Formación, tienen poco que ver con los conocimientos y prácticas profesionales. En este caso los estudiantes suelen deslumbrarse por la realidad, y cuando se reincorporan de nuevo a la actividad académica, comienzan a desechar, por considerarla menos importante, la necesidad de ciertos conocimientos que fundamenten el trabajo práctico.

\section{Formadores de profesores universitarios y profesores} supervisores de aula

Derivada de la laguna de los dos mundos sigue constatándose una enorme distancia y falta de diálogo y compromiso compartido entre los principales formadores que influyen en los profesores en formación: los formadores universitarios y los profesores de aula (supervisores, colaboradores, tutores o mentores). Como mostramos en otro trabajo (Marcelo \& Estebaranz, 1998), las relaciones entre formadores universitarios y escolares pueden ser variadas pero predominan los modelos que podríamos denominar "cada cual en lo suyo". Si queremos mejorar la formación inicial del profesorado debemos hacer que haya una mayor coincidencia entre los modelos y prácticas docentes que se les presentan a los profesores en formación.

\section{Conocimiento disciplinar y conocimiento pedagógico}

Esta yuxtaposición es también tradicional. La respuesta a la pregunta ¿̇cuál es el conocimiento que los profesores deben de poseer? no ha tenido ni tiene una única respuesta. Para muchos sistemas educativos lo importante es que los profesores conozcan la materia que enseñan. Esta afirmación con la que difícilmente podríamos estar en desacuerdo tiene sus puntos oscuros. ¿Hablamos de almacenar información o de comprender en profundidad?

Una línea de investigación y práctica ha venido a ofrecer algunos puentes significativos entre ambos elementos: me refiero a los trabajos sobre "Conocimiento Didáctico del Contenido" al que me he referido an- 
teriormente. El Conocimiento del Contenido incluye diferentes componentes, de los cuales dos son los más representativos: conocimiento sintáctico y sustantivo. El Conocimiento Sustantivo se constituye con la información, las ideas y los tópicos a conocer, es decir, el cuerpo de conocimientos generales de una materia, los conceptos específicos, definiciones, convenciones, y procedimientos. Este conocimiento es importante en la medida en que determina lo que los profesores van a enseñar y desde qué perspectiva lo harán. El Conocimiento Didáctico del Contenido aparece como un elemento central de los saberes del formador. Representa la combinación adecuada entre el conocimiento de la materia a enseñar y el conocimiento pedagógico y didáctico referido a cómo enseñarla. En los últimos años, se ha venido trabajando en diferentes contextos educativos para clarificar cuáles son los componentes de este tipo de conocimiento profesional de la enseñanza. El Conocimiento Didáctico del Contenido, como línea de investigación, representa la confluencia de esfuerzos de investigadores didácticos con investigadores de materias específicas preocupados por la formación del profesorado. El Conocimiento Didáctico del Contenido nos dirige a un debate en relación con la forma de organización y de representación del conocimiento, a través de analogías y metáforas. Plantea la necesidad de que los profesores en formación adquieran un conocimiento experto del contenido a enseñar, para que puedan desarrollar una enseñanza que propicie la comprensión de los alumnos.

\section{Teoría y práctica: desvalorización de la teoría y adoración de la} práctica

Quizás como resultado de un movimiento pendular, se ha producido en la formación del profesorado una inclinación, desde mi punto de vista excesiva, hacia lo que se ha considerado como "la práctica", asumiendo que es el contacto con la práctica el que hace al profesor. De esta forma, cualquier aproximación que pretenda ayudar a conceptuar y comprender al profesorado las experiencias y la complejidad del acto de enseñar se rechaza como "teórico". Decía Perkins que "comprender es la habilidad de pensar y actuar con flexibilidad a partir de lo que uno ya sabe". (1999, p. 70). Para ayudar a los profesores a comprender qué ocurre en el aula y las razones de la conducta de los alumnos y de los formadores se requieren marcos conceptuales necesarios para que cada profesor 
desarrolle su propia identidad. Otra cosa es asumir la práctica como un valor en sí mismo, algo que creo no ayuda a mejorar la formación del profesorado.

Cochran-Smith y Lytle (1999), han reflexionado sobre las relaciones entre conocimiento y práctica en la formación del profesorado y nos plantean que las cosas pueden tener diferentes puntos de vista. Así, diferencian entre: Conocimiento para la práctica: Esta primera concepción entiende en que la relación entre conocimiento y práctica es aquella en que el conocimiento sirve para organizar la práctica, y por ello, conocer más (contenidos, teorías educativas, estrategias instruccionales) conduce de forma más o menos directa a una práctica más eficaz. El conocimiento para enseñar es un conocimiento formal, que se deriva de la investigación universitaria, y es al que se refieren los teóricos cuando se habla de que la enseñanza ha generado un cuerpo de conocimiento diferente al conocimiento común. La práctica, desde esta perspectiva, tiene mucho que ver con la aplicación del conocimiento formal a las situaciones prácticas.

Por otra parte, el Conocimiento en la práctica pone el énfasis de la investigación sobre aprender a enseñar ha sido la búsqueda del conocimiento en la acción. Se ha estimado que lo que los profesores conocen está implícito en la práctica, en la reflexión sobre la práctica, en la indagación práctica y en la narrativa de esa práctica. Un supuesto de esta tendencia es que la enseñanza es una actividad incierta y espontánea, contextualizada y construido en respuesta a las particularidades de la vida diaria en las escuelas y las clases. El conocimiento está situado en la acción, en las decisiones y juicios que toman los profesores. Este conocimiento se adquiere mediante la experiencia y la deliberación y los profesores aprenden cuando tienen oportunidad de reflexionar sobre lo que hacen.

Por último, el Conocimiento de la práctica se incluye dentro de la línea de investigación cualitativa, pero cercana al movimiento denominado del profesor como investigador. La idea de la que parte es que en la enseñanza no tiene sentido hablar de un conocimiento formal y otro conocimiento práctico, sino que el conocimiento se construye colectivamente dentro de comunidades locales, formadas por profesores trabajando en proyectos de desarrollo de la escuela, de formación o de indagación colaborativa (COCHRAN-SMITH; LYTLE, 1999). 


\section{Tradición e innovación}

¿Mantener las esencias o cambiar? Un buen dilema que observamos siempre que analizamos una reforma en los planes y programas de formación del profesorado. Podemos decir que gran parte de las reformas que se han ido introduciendo en la formación inicial del profesorado se han movido más en el terreno de la tradición que de la innovación. Las experiencias innovadoras en formación de profesores, especialmente en formación inicial suelen ser ocasionales y su duración en el tiempo está limitada a la persistencia de los recursos o las personas que la han puesto en marcha. Si como decía Bereiter (2002) la innovación la fuerza que impulsa a la sociedad del conocimiento, ésta debería de estar presente en unas instituciones llamadas a formar a los profesionales del conocimiento que son los docentes.

\section{Homogeneidad y diversidad: profesores homogéneos para un alumnado diverso}

Uno de los grandes cambios que se están produciendo en nuestras sociedades es el aumento de la movilidad e inmigración. Los países europeos están recibiendo una gran cantidad de personas procedentes de otros continentes. Estos cambios demográficos están teniendo ya una presencia evidente en nuestras escuelas. Hay clases en las que es fácil encontrar alumnos de diez países diferentes. Es ésta una nueva realidad para la que el profesorado no se encuentra preparado. Una de las críticas a los programas de formación del profesorado es su escasa adaptación a los cambios que se producen en relación con la diversidad de los estudiantes en las aulas. De manera gráfica, Ladson-Billing afirmaba que "se sigue formando a los profesores para enseñar en escuelas ideales con niños blancos, monolingües, de clase media y de familias con dos padres” (1998, p. 87). A esta crítica se unen Grant y Wieczorek (2000) al destacar la ausencia de un análisis crítico sobre los aspectos y condicionamientos sociales del conocimiento, que pueden encontrarse en temas como la raza, clase social, género y poder, así como los elementos históricos y políticos ligados a la producción de conocimiento. La diversidad es una realidad que debe entrar en la formación del profesorado para ayudar a los docentes en formación, así como en ejercicio a enfrentar su tarea de una forma más positiva. 
La formación docente en la sociedad del conocimiento y la información

\section{Enseñanza y aprendizaje}

"Yo enseño pero los alumnos no aprenden". Éste vendría a ser el resumen de la situación por la que atraviesan muchos profesores. Quizás la formación del profesorado, así como la investigación, se ha centrado tradicionalmente en la figura del profesor en interacción con un grupo más o menos numeroso de alumnos intentando transmitirles conocimiento de manera oral. Ya sé que ésta es una imagen demasiado radical pero me sirve para plantear que "en la sociedad del conocimiento" debemos de prestar atención no sólo a lo que los profesores hacen, sino a lo que los alumnos aprenden. La apuesta por una enseñanza centrada en la actividad de aprendizaje de los alumnos tiene sus fundamentos científicos. Duffy, Dueber y Hawley (1998, p. 51) afirmaban que

Existe actualmente un movimiento muy fuerte en educación que se aleja del modelo didáctico predominante y que se encamina hacia un modelo centrado en el que aprende, donde las actividades de aprendizaje implican a los alumnos en la indagación y resolución de problemas, normalmente en un espacio colaborativo".

Estamos avanzando rápidamente modelos de aprendizaje alternativos que desde un punto de vista genérico se denominan como constructivistas en los que el énfasis se sitúa en la orientación y apoyo a los estudiantes en la medida en que éstos aprenden a construir su conocimiento y comprensión de la cultura y la comunidad a la que pertenecen (BONK; CUNNINGHAM, 1998). El concepto de ambientes de aprendizaje constructivistas ha ido ganando terreno entre las personas que nos dedicamos al diseño de acciones de enseñanza y formación a través de Internet. Wilson $(1996$, p. 3) decía que

Un ambiente de aprendizaje es un lugar donde las personas pueden utilizar recursos para dar sentido a cosas y a soluciones significativas a problemas. Al añadir el término constructivista al final se pone énfasis en la importancia de lo significativo, de actividades auténticas que ayuden a los alumnos a construir conocimiento y desarrollar destrezas relevantes para resolver problema. 
De esta manera, pensar y utilizar el concepto de "Ambiente de Aprendizaje" como metáfora supone en un espacio donde ocurre el aprendizaje. Un espacio que puede ser real o virtual, pero en cualquiera de las situaciones debería atender de manera especial a la persona que aprende, la situación o espacio donde actúa, interacciona y aprende el alumno, y la utilización de herramientas y medios que faciliten el aprendizaje. Otra forma de definirlo sería: "un lugar donde los alumnos pueden trabajar juntos y apoyarse unos a otros en la medida en que utilizan una variedad de herramientas y recursos de información en su búsqueda de objetivos de aprendizaje y en la realización de actividades de resolución de problemas". (WILSON, 1996).

\section{Formación inicial y formación continua}

¿Qué decir del divorcio entre la formación inicial y la formación continua? ¿Qué hay de ese eslabón perdido que representan los programas de inserción profesional (inducción) para los profesores principiantes? La inserción profesional en la enseñanza, es el periodo de tiempo que abarca los primeros años, en los cuales los profesores han de realizar la transición desde estudiantes a profesores. Es un periodo de tensiones y aprendizajes intensivos en contextos generalmente desconocidos y durante el cual los profesores principiantes deben adquirir conocimiento profesional además de conseguir mantener un cierto equilibrio personal. Es éste el concepto de inserción que asume Vonk, autor holandés con una década de investigaciones centradas en éste ámbito: "definimos la inserción como la transición desde profesor en formación hasta llegar a ser un profesional autónomo. La inserción se puede entender mejor como una parte de un continuo en el proceso de desarrollo profesional de los profesores" (1996, p. 115).

Conviene insistir en esta idea de que el periodo de inserción es un periodo diferenciado en el camino de convertirse en profesor. No es un salto en el vacío entre la formación inicial y la formación continua sino que tiene un carácter distintivo y determinante para conseguir un desarrollo profesional coherente y evolutivo (BRITTON, PAINE, PIMM; RAIZEN, 2002). El periodo de inserción y las actividades propias que le acompañan varían mucho entre los países. En algunos casos se reducen a actividades burocráticas y formales. En otros casos, como veremos más adelante configuran toda una propuesta de programa de formación cuya intención es asegurar que los profesores 
La formación docente en la sociedad del conocimiento y la información

entren en la enseñanza acompañados por otros que pueden ayudarle.

Los profesores principiantes tienen, según Feiman (2001) dos tareas que cumplir: deben enseñar y deben aprender a enseñar. Independientemente de la calidad del programa de formación inicial que hayan cursado, hay algunas cosas que sólo se aprenden en la práctica y ello repercute en que este primer año sea un año de supervivencia, descubrimiento, adaptación, aprendizaje y transición. Las principales tareas con que se enfrentan los profesores principiantes son: adquirir conocimientos sobre los estudiantes, el currículo y el contexto escolar; diseñar adecuadamente el currículo y la enseñanza; comenzar a desarrollar un repertorio docente que les permita sobrevivir como profesor; crear una comunidad de aprendizaje en el aula, y continuar desarrollando una identidad profesional. Y el problema es que esto deben hacerlo en general cargados con las mismas responsabilidades que los profesores más experimentados (MARCELO, 1999a).

El periodo de inserción profesional se configura como un momento importante en la trayectoria del futuro profesor. Un periodo importante porque los profesores deben realizar la transición de estudiantes a profesores, por ello surgen dudas, tensiones, debiendo adquirir un adecuado conocimiento y competencia profesional en un breve período de tiempo. En este primer año los profesores son principiantes, y en muchos casos, incluso en su segundo y tercer año pueden todavía estar luchando para establecer su propia identidad personal y profesional. Atender a los profesores principiantes resulta fundamental para poder tener la esperanza de que el profesorado asume el aprendizaje a lo largo de la vida como un compromiso profesional.

\section{Aprendizaje formal e informal}

Si algo caracteriza a la sociedad del conocimiento es la accesibilidad de éste a todos los ciudadanos. Pero el conocimiento no tiene igual reconocimiento por la sociedad dependiendo de donde éste proceda. Antes hablábamos de la dicotomía entre teoría y práctica y ahora podemos hablar de aprendizaje formal e informal. El aprendizaje informal es un tipo de aprendizaje que contrasta con el aprendizaje formal. Hager (2001) establece las diferencias en los siguientes términos:

El formador controla el aprendizaje formal mientras que es el alumno el que controla el aprendizaje informal: el aprendizaje formal 
se planifica mientras que el informal no.

El aprendizaje formal se desarrolla en instituciones educativas, en el trabajo y es ampliamente predecible. El aprendizaje informal no es predecible y no posee un currículo formal.

Tanto en las instituciones educativas como en la formación, el aprendizaje es explícito: se espera que el que ha asistido a formación sea capaz de demostrarlo mediante exámenes escritos, respuestas orales, etc. El aprendizaje informal generalmente es implícito, y en general el aprendiz no es consciente de lo que sabe, aunque sea consciente de los resultados de ese aprendizaje.

En el aprendizaje formal se pone énfasis en la enseñanza, en el contenido y la estructura de lo que va a ser enseñado, mientras que en el aprendizaje informal el énfasis es en el que aprende.

En el aprendizaje informal el énfasis recae en los alumnos como individuos o en el aprendizaje individual, mientras que el aprendizaje informal a menudo es colaborativo.

El aprendizaje formal es descontextualizado, mientras que el aprendizaje informal es de naturaleza contextualizada

El aprendizaje formal toma forma en términos de teoría (o conocimiento) y después práctica (aplicación de la teoría), mientras que el aprendizaje informal tiene que ver más con conocer cómo se hacen las cosas.

Nos ha llamando la atención a partir de la lectura del sugerente libro que sobre la Ética del Hacker ha publicado el filósofo finlandés Pekka Himanen (2001). Para este autor, el paradigma del aprendizaje en la sociedad del conocimiento tiene mucho que ver con la forma como los hackers aprenden. Recordemos que nos referimos al hacker como una persona con conocimientos informáticos y que desarrolla por sí mismo y en colaboración con otros, alternativas y desarrollos informáticos que desafían claramente a las grandes firmas comerciales de este sector.

Pues bien, plantea Himanen, tomando como ejemplo a Linus Torval, autor del sistema operativo Linux, que el aprendizaje, en la sociedad del conocimiento, tiene que estar asociado con la pasión, con el interés por lo desconocido, por las preguntas más que por las respuestas, por el apoyo de otros que conocen, por la resolución de problemas de manera colaborativa. Ese modelo de aprendizaje en el que lo que agrupa a las personas que aprenden no es la edad sino el problema a resolver, algo parecido a la Academia de Platón. Pero, 
La formación docente en la sociedad del conocimiento y la información

como comenta Himanen, "La ironía es que la actual academia tiende a reproducir la estructura de aprendizaje emisor-receptor propia de los monasterios. La ironía generalmente se amplía cuando la academia construye una 'universidad virtual': el resultado es una escuela monacal computerizada". (2001, p. 76).

\section{Aislamiento en el aula y la sociedad en red}

Hoy día convivimos con un nuevo dilema: si por una parte gran parte del profesorado tiene la oportunidad de acceder a fuentes de conocimiento, materiales, experiencias, así como implicarse en redes de profesores (Marcelo, 2002b), se sigue manteniendo en las aulas un de los principios de la cultura profesional docente: el aislamiento. El desarrollo y generalización de redes de profesores, la posibilidad de aprender con otros a la distancia, la creación de escenarios abiertos y distribuidos que actualmente están siendo posibles gracias a las nuevas tecnologías de la información y la comunicación, están facilitando la visibilidad de esa forma de aprendizaje que hemos llamando informal. Y este hecho está removiendo los tranquilos cimientos de las instituciones formales de acreditación.

\section{PARA CONCLUIR}

¿Vemos el vaso medio lleno o medio vacío? Hay razones a favor de cualquiera de las opciones. Podemos tener la sensación de que vamos avanzando en el conocimiento sobre el aprender a enseñar, pero si miramos a nuestro alrededor vemos que muchas de las prácticas más tradicionales permanecen. Me llamó mucho la atención un artículo de Labaree $(1998$, p. 9) en el que, después de analizar el tipo de conocimiento sobre el que trabajamos los investigadores educativos, concluía con una frase que me permito reproducir. Decía que

Un problema que el conocimiento educativo plantea a aquellos que buscan producirlo es que a menudo les deja con la sensación de estar perpetuamente luchando por avanzar hacia ninguna parte. Si Sísifo fuera universitario, su campo sería la educación. $\mathrm{Al}$ final de una larga y distinguida carrera, muchos investigadores en edad de jubilación suelen encontrar que aún se 
encuentran trabajando en los mismos problemas que abordaban al comienzo de su carrera.

No es que uno se encuentre ya al final de su carrera, pero sí que cuenta con el tiempo suficiente como para comprobar que muchos de los temas que configuran la agenda de la reforma de la formación del profesorado siguen estando presentes, como si el tiempo, las investigaciones o la práctica no hubiera permitido generar avances sustanciales. Esos temas nos siguen resultando familiares. ¿Será que tiene que ser así?

\section{REFERÊNCIAS}

BEREITER, C. Education and mind in the knowledge age. New Jersey: Lawrence Erlbaum, 2002.

BEREITER, C.; Scardamalia, M. Educational relevance of the study of expertise. Interchange, v. 17, n. 2, p. 10-19, 1986.

BERLINER, D. In pursuit of the expert pedagogue. Educational Researcher, v. 15, n. 7, p. 5-13, 1986.

BERLINER, D. C. A personal response to those who bash teacher education. Journal of Teacher Education, v. 51, n. 5, p. 358-371, 2000.

BONK, C.; CUNNINGHAM, D. Searching for learner-centered, constructivism, and sociocultural components of collaborative educational learning tools. In: BONK, C.; KING, K. (Ed.). Electronic Collaborators. New Jersey: Lawrence Erlbaum Ass, 1998. p. 25-50.

BRANSFORD, J.; DARLING-HAMMOND, L.; LEPAGE, P. Introduction. In: DARLING-HAMMOND, L.; BRANSFORD, J. (Ed.). Preparing teachers for a changing world.. S. Francisco: Jossey Bass, 2005. p.1-39.

BRANSFORD, J. et al. Theories of learning and their roles in teaching. In: DARLING-HAMMOND, L.; BRANSFORD, J. (Ed.). Preparing teachers for a changing world. S. Francisco: Jossey Bass, 2005. p. 40-87.

BRITTON, E. et al. Comprehensive teacher induction. Dordrecht: Kluwer Academic Press, 2002.

BULLOUGH, R. Becoming a teacher. In: BILDDLE, B. (Ed.). Internatinal handbook of teacher and teaching. London: Kluwer., 1998. p. 79-134.

COCHRAN-SMITH, M.; FRIES, K. The AERA panel on research and teacher 
La formación docente en la sociedad del conocimiento y la información

education: context and goals. In: COCHRAN-SMITH, M.; ZEICHNER, K. (Ed.). Studying teacher education: the report of the AERA Panel on research and teacher education. New Jersey: Lawrence Erlbaum Associates, 2005. p.37-68.

COCHRAN-SMITH, M.; LYTLE, S. The teacher research movement: a decade later. Educational Researcher, v. 28, n. 7, p.15-25, 1999.

COCHRAN-SMITH, M.; ZEICHNER, K. Studying teacher education: the report of the AERA Panel on research and teacher education. New Jersey: awrence Erlbaum Ass, 2005.

DARLING-HAMMOND, L. Teacher quality and student achievement: a review of state policy evidence. [Electronic Version]. Educational Policy Analysis Archives, v. 8, n. 1. 2000. Disponível em: http://epaa.asu.edu/epaa/v8n1/ . Acesso em: 10 ago. 2006.

DUFFY, T.; DUEBER, B.; HAWLEY, C. Critical thinking in a distributed environment: A pedagogical base for the design of conferencing systems. In: BONK, C. KING, K. (Ed.). Electronic Collaborators. New Jersey: Lawrence Erlbaum Ass., 1998. p.51-78.

FEIMAN-NEMSER, S. From preparation to practice: designing a continuum to strengthen and sustain teaching. Teachers College Record, v. 103, n. 6, p.1013-1055, 2001.

FEIMAN-NEMSER, S.; BUCHMAN, M. Lagunas de las prácticas de enseñanza de los programas de formación del profesorado. In: VILLAR ANGULO, L. M. (Ed.). Conocimiento, creencias y teorías de los profesores. Alicante: Marfil, 1988. p.301-314.

FENSTERMACHER, G. The knower and the known: the nature of knowledge in research on teaching. In: DARLING-HAMMOND, L. (Ed.). Review of Research in Education. Washington: American Educational Research Association. 1994. p.3-56.

GRANT, C. A.; WIECZOREK, K. Teacher education and knowledge in "the Knowledge Society": the need for social moorings in our multicultural schools. Teacher College Record, v. 102, n. 5, p. 913-935, 2000.

HAGER, P. Lifelong learning and the contribution of informal learning. In: ASPIN, D. et al. (Ed.). International Handbook of Lifelong Learning. London: Kluwer, 2001. p.79-92.

HIMANEN, P. The hacker ethic. New York: Random House, 2001.

LABAREE, D. Educational researchers: livingwith a lesser form of knowledge. Educational Researcher, v. 27, n. 8, p. 4-12, 1998. 
LADSON-BILLING, G. Preparing teachers for diversity. In: Darling-Hammond, L.; SYKES, G. (Ed.). Teaching as a learning professional. Handbook of Policy and Practice. S.Francisco: Jossey-Bass, 1998. p.86-121.

LORTIE, D. School teachers: a sociological study. Chicago: University of Chicago Press, 1975.

MARCELO, C. Estudio sobre estrategias de inserción profesional en Europa. Revista Iberoamericana de Educación, n. 19, p. 101-144, 1999a. lona: EUB, 1999b.

Formación de profesores para el cambio educativo. Barce-

- Aprender a enseñar para la sociedad del conocimiento.

[Electronic Version]. Educational Policy Analysis Archives, 10. 2002a. Disponível em: http://epaa.asu.edu/epaa/v10n35/. Acesso em: 10 ago. 2006.

E-Learning-Teleformación. Diseño, desarrollo y evaluación de la formación a través de internet. Barcelona: Gestión 2000, 2002b.

.; ESTEBARANZ, A. Modelos de colaboración entre la universidad y las escuelas en la formación del profesorado. Revista de Educación, 317, 97-120, 1998.

OCDE. Teachers matter: attracting, developing and retaining effective teachers. Paris: OCDE, 2005.

PAJARES, M. F. Teachers' beliefs and educational research: cleanning up a messy construct. Review of Educational Research, v. 62, n. 3, p. 307-332, 1992.

PERKINS, D. ¿Qué es la comprensión? In: STONE WISKE, M. (Ed.). La enseñanza para la comprensión. Buenos Aires: Paidos, 1999.

PUTNAM, R.; BORKO, H. Teachers learning: implications of new views of cognition. In: BIDDLE, B. et al. (Ed.). International Handbook of Teachers and Teaching. London: Kluwer, 1998. p. 1223-1296.

RICHARDSON, V. Handbook of Research on Teaching. 4. ed. New York: Americam Educational Research Association, 2001.

RICHARDSON, V.; PLACIER, P. Teacher change. In: RICHARDSON, V. (Ed.). Handbook of research on teaching. 4. ed. New York: American Educational Research Association, 2001. p.905-947.

SALOMON, G. The changing role of the teachers: from information transmitter to orchestrator of learning. In: OSER, F.; DICK, A.; PATRY, J. (Eds.). Effective and responsible teaching: the new synthesis. New York: Jossey-Bass, 1992. p.35-49. 
La formación docente en la sociedad del conocimiento y la información

SENGE, P. La quinta disciplina: el arte y la práctica de la organización abierta al aprendizaje. Barcelona: Granica, 1992.

STASZ, C. Assessing skills for work: two Perspectives: Oxford Economic Papers, 2000.

STONE WISKE, M. La enseñanza para la comprensión. Buenos Aires Paidos, 1999.

VAILLANT, D.; MARCELO, C. Las tareas del formador. Malaga: Aljibe, 2001.

VONK, J. H. C. A Knowledge base for mentors of beginning teachers: results of a dutch experience. In: MCBRIDGE, R. (Ed.). Teacher Education Policy. London: Falmer Press, 1996. p. 112-134.

WIDEEN, M.; MAYER-SMITH, J.; MOON, B. A critical analysis of the research on learning to teach: making the case for an ecological perspective on inquiry. Review of Educational Reseach, n.68, p. 130-178, 1998.

WILSON, B. What is a constructivist learning environment? In: WILSON, B. (Ed.). Constructivist learning environments. Englewood Cliffs: Educational Technology Publications, 1996. p.3-7.

WILSON, S.; BERNE, J. Teacher learning and the acquisition of professional knowledge: an examination of research on contemporary professional development. In: IRAN-NEJAD, A.; PERSON, P.. (Eds.). Review of Research in Educaton. Washington: Americam Educational Research Association, 1999. p. 173-209.

ZEICHNER, K. (1999). The new scholarship in teacher education. Educational Researcher, v. 28, n. 9, p. 4-15. 1999.

Encaminhado em: 04/07

Aceito em: 04/07 